OPEN ACCESS

Edited by: Olaf Van Tellingen,

The Netherlands Cancer Institute (NKI), Netherlands

Reviewed by:

Cornelis Johannes Forrendinis Van Noorden,

Academic Medical Center, Netherlands

Paul Walker,

Université de Genève, Switzerland

*Correspondence:

Rosa Maria Moresco rosa.moresco@unimib.it

Specialty section:

This article was submitted to Neuro-Oncology and Neurosurgical Oncology, a section of the journal

Frontiers in Oncology

Received: 04 February 2021 Accepted: 12 April 2021 Published: 03 May 2021

Citation:

Valtorta S, Lo Dico A, Raccagni I, Martelli C, Pieri V, Rainone P, Todde S, Zinnhardt B, De Bernardi E, Coliva A, Politi LS, Viel T, Jacobs AH, Galli $R$, Ottobrini L, Vaira $V$ and Moresco RM (2021) Imaging Metformin Efficacy as Add-On Therapy in Cells and Mouse Models of Human EGFR Glioblastoma.

Front. Oncol. 11:664149. doi: 10.3389/fonc.2021.664149

\section{Imaging Metformin Efficacy as Add-On Therapy in Cells and Mouse Models of Human EGFR Glioblastoma}

\author{
Silvia Valtorta ${ }^{1,2,3}$, Alessia Lo Dico ${ }^{4}$, Isabella Raccagni ${ }^{2,3,5}$, Cristina Martelli ${ }^{4}$, Valentina Pieri ${ }^{6}$, \\ Paolo Rainone ${ }^{1,3}$, Sergio Todde ${ }^{1,2}$, Bastian Zinnhardt ${ }^{7,8}$, Elisabetta De Bernardi ${ }^{1}$, \\ Angela Coliva ${ }^{3}$, Letterio S. Politi ${ }^{9,10}$, Thomas Viel ${ }^{11}$, Andreas H. Jacobs ${ }^{7}$, Rossella Galli ${ }^{6}$, \\ Luisa Ottobrini ${ }^{2,4}$, Valentina Vaira ${ }^{4,12}$ and Rosa Maria Moresco ${ }^{1,2,3^{*}}$ \\ ${ }^{1}$ Department of Medicine and Surgery and Tecnomed Foundation, University of Milano - Bicocca, Monza, Italy, 2 Institute of \\ Molecular Bioimaging and Physiology, National Research Council (IBFM-CNR), Segrate, Italy, ${ }^{3}$ Nuclear Medicine \\ Department, IRCCS San Raffaele Scientific Institute, Milan, Italy, ${ }^{4}$ Department of Pathophysiology and Transplantation \\ (DEPT), University of Milan, Milan, Italy, ${ }^{5}$ SYSBIO Centre of Systems Biology ISBE.ITALY, University of Milano - Bicocca, \\ Milan, Italy, ${ }^{\circ}$ Neural Stem Cell Biology Unit, Division of Neuroscience, IRCCS San Raffaele Scientific Institute, Milan, Italy, \\ ${ }^{7}$ European Institute for Molecular Imaging (EIMI), University of Münster, Münster, Germany, ${ }^{8}$ Department of Nuclear \\ Medicine, University Hospital Münster, Münster, Germany, ${ }^{9}$ Department of Biomedical Sciences, Humanitas University, \\ Rozzano, Italy, ${ }^{10}$ Department of Neuroradiology, Humanitas Clinical and Research Center IRCCS, Rozzano, Italy, \\ 11 PARCC, INSERM, Université de Paris, Paris, France, ${ }^{12}$ Division of Pathology, Fondazione IRCCS Ca' Granda Ospedale \\ Maggiore Policlinico, Milan, Italy
}

Glioblastoma (GBM) is a highly aggressive tumor of the brain. Despite the efforts, response to current therapies is poor and 2-years survival rate ranging from 6-12\%. Here, we evaluated the preclinical efficacy of Metformin (MET) as add-on therapy to Temozolomide (TMZ) and the ability of $\left[{ }^{18} \mathrm{~F}\right] \mathrm{FLT}$ (activity of thymidine kinase 1 related to cell proliferation) and $\left[{ }^{18} \mathrm{~F}\right] \mathrm{VC} 701$ (translocator protein, TSPO) Positron Emission Tomography (PET) radiotracers to predict tumor response to therapy. Indeed, TSPO is expressed on the outer mitochondrial membrane of activated microglia/macrophages, tumor cells, astrocytes and endothelial cells. TMZ-sensitive (Gli36 LEGFR-1 and L0627) or -resistant (Gli36 EEGFR-2) GBM cell lines representative of classical molecular subtype were tested in vitro and in vivo in orthotopic mouse models. Our results indicate that in vitro, MET increased the efficacy of TMZ on TMZ-sensitive and on TMZ-resistant cells by deregulating the balance between pro-survival (bcl2) and pro-apoptotic (bax/bad) Bclfamily members and promoting early apoptosis in both Gli36 $\Delta$ EGFR-1 and Gli36 $\Delta$ EGFR-2 cells. In vivo, MET add-on significantly extended the median survival of tumor-bearing mice compared to TMZ-treated ones and reduced the rate of recurrence in the TMZsensitive models. PET studies with the cell proliferation radiopharmaceutical $\left.{ }^{18} \mathrm{~F}\right] \mathrm{FLT}$ performed at early time during treatment were able to distinguish responder from nonresponder to TMZ but not to predict the duration of the effect. On the contrary, $\left[{ }^{18} \mathrm{~F}\right] \mathrm{VC} 701$ uptake was reduced only in mice treated with MET plus TMZ and levels of uptake negatively correlated with animals' survival. Overall, our data showed that MET addition improved TMZ efficacy in GBM preclinical models representative of classical molecular 
subtype increasing survival time and reducing tumor relapsing rate. Finally, results from PET imaging suggest that the reduction of cell proliferation represents a common mechanism of $\mathrm{TMZ}$ and combined treatment, whereas only the last was able to reduce TSPO. This reduction was associated with the duration of treatment response. TSPOligand may be used as a complementary molecular imaging marker to predict tumor microenvironment related treatment effects.

Keywords: GBM - glioblastoma multiforme, metformin, inflammation, PET imaging, [18F]FLT, EGFR - epidermal growth factor receptor, TSPO

\section{INTRODUCTION}

Glioblastoma (GBM) represents the most common and aggressive malignant brain neoplasm in adults with no effective treatments. Surgical resection and concomitant radiotherapy followed by adjuvant Temozolomide (TMZ) (Stupp protocol) represent the gold standard for GBM treatment (1). Nevertheless, resistance to TMZ and/or disease progression invariably occur in GBM independently of O6-methylguanine-DNA methyltransferase (MGMT) presence (2) leading to a poor clinical outcome and a median overall-survival of 14.6 months. For this reason, novel treatment approaches for GBM represent an unmet medical need (3). Despite the efforts, the majority of new therapeutic strategies proposed, including targeted-based therapy, showed limited efficacy in clinical trials. The lack of success of existing or newly developed therapy is based on several factors, including the biological complexity and the clonal heterogeneity of GBM. A common hallmark of GBM is represented by an aberrant metabolic phenotype characterized by increased glucose demand and aerobic glycolysis (the so-called Warburg effect) (4). In addition, many of the oncogenes and tumor suppressor proteins, commonly mutated in GBM, regulate cancer metabolism leading to an increased glucose uptake, the switch to the Warburg effect, de novo lipogenesis and other alternative metabolic pathways. For the reason above, targeting tumor metabolism represents an attractive therapeutic strategy for $\operatorname{GBM}(5,6)$ particularly using combined strategies (7). The oral antidiabetic Metformin (MET), that modulates 5' AMP-activated protein kinase (AMPK) and mitochondrial functions, showed promising in vitro and in vivo results in different types of cancer, including GBM (8-10). MET was initially proposed as a single regimen against glioma-initiating stem cells, however, we and other groups demonstrated that MET is synergic with TMZ and is able to revert TMZ resistance in some mouse models of GBM (11-13). Another negative hallmark of glioma is represented by the high variability of molecular phenotypes. Using an unsupervised hierarchical clustering analysis, Verhaak et al. classified GBM in four molecular subtypes, named Classical, Mesenchymal, Neural and Proneural (14). The four subtypes differ for rate of progression, response to chemotherapy and for molecular signature. The Epidermal Growth Factor Receptor (EGFR) amplification or mutation is present in approximately $57 \%$ of tumors, particularly the classical subtype (15). Approximately 50\% of tumors carrying EGFR amplification present a specific highly oncogenic and constitutively activated mutant (EGFRvIII, also known as EGFR type III, de2-7, $\triangle$ EGFR) (16). Overall, the hyper-activated EGFR phenotype favors treatment resistance and poor clinical outcome (17).

Despite the major role in cell growth, the clinical efficacy of EGFR tyrosine kinase inhibitors was poor. Interestingly, Ciaglia et al. showed that activation of the metabolic sensor AMPK through the administration of N6-isopentenyladenosine (iPA) inhibited the in vivo growth of GBM tumors, with markedly enhanced efficacy in cells with higher levels of EGFR expression/ activity (18). Another important point is that EGFR favors a highly inflammatory microenvironment in $\operatorname{GBM}(19,20)$. Although the role of inflammation in glioma is not completely understood, several studies on immune check-point inhibitors suggest a link between inflammation and tumor progression or relapsing in GBM (21). Indeed, recent data showed the ability of MET of targeting the inflammatory tumor microenvironment, contributing to reduction of tumor mass and of cancer related M2 macrophage polarization (22).

For the reasons above, the primary objective of our study was to evaluate the effect of MET used in combination with TMZ on EGFR mutation (d2-7) carrying GBM models sensitive and resistant to TMZ and on patient-derived EGFR amplified Cancer Stem Cell line. Furthermore, we aimed to evaluate the potential use of in vivo Positron Emission Tomography (PET) molecular imaging to predict drug effects. For this purpose we measured at early time after treatment the uptake of $\left[{ }^{18} \mathrm{~F}\right] \mathrm{FLT}$ and $\left[{ }^{18} \mathrm{~F}\right]$ VC701 radiopharmaceuticals targeting thymidine kinase 1 (TK1) and Translocator Protein $18 \mathrm{kDa}$ (TSPO) which are receptors associated with glioma malignancy. Despite its presence has been described also in tumors, increased levels of TSPO are associated with the presence of clusters of microglial/macrophage cells with an activated phenotype (23). For this reason, TSPO ligands, including $\left[{ }^{18} \mathrm{~F}\right] \mathrm{VC701}$ are used to image the inflammatory reaction present during tumor development and the relative modulation induced by drugs $(24,25)$. Finally, to investigate therapy effects on tumor proliferation and inflammation markers, Ki67 and Ibal were evaluated post mortem by immunohistochemistry (IHC).

\section{MATERIALS AND METHODS}

\section{Cell Culture}

Sensitive (Gli36 $\Delta$ EGFR-1 and L0627) or resistant (Gli36 $\Delta E G F R-$ 2) to TMZ GBM cells representative of classical subtype were used in this study. Human GBM Gli36 2 EGFR cells (kind gift of Dr. David Louis, Molecular Neurooncology Laboratory, MGH, 
Boston, MA) $(26,27)$ carry a mutant epidermal growth factor receptor ( $\Delta 2-7$, EGFR). Gli36 $\Delta$ EGFR cells were called Gli36 $\Delta$ EGFR-1 to underline the sensitivity to Temozolomide (TMZ) treatment compared to the cell line obtained after treatment with sub-lethal doses of TMZ (50 $\mu \mathrm{M}$ of TMZ for 1 month) defined as Gli36 $\Delta$ EGFR-2 (28). Cells were maintained in Dulbecco's Modified Eagle Medium (DMEM) with high glucose supplemented with $10 \%$ heat-inactivated Foetal Bovine Serum (FBS), and $50 \mathrm{IU} / \mathrm{ml}$ Penicillin/Streptomycin (P/S), $2 \mathrm{mM}$ glutamine (all Euroclone, UK) at $37^{\circ} \mathrm{C}$ in a $5 \% \mathrm{CO}_{2} / 95 \%$ air atmosphere. L0627 GBM CSCs, established at the Neural Stem Cell Biology Unit, San Raffaele Scientific Institute, Milan, Italy and validated in Narayanan et al. (29) and Mazzoleni et al. (30) were cultured under the conditions of the NeuroSphere Assay (NSA) (31). GBM cells were carefully cultured and monitored and in vitro displayed a typical growth pattern and phenotype.

\section{Treatments Assay}

10,000 cells $/ \mathrm{cm}^{2}$ Gli36 $\Delta$ EGFR-1 and Gli36 $\Delta$ EGFR-2 cells were exposed to different concentrations of $\operatorname{TMZ}(0.1,1,5,10,25,50$, 100, $200 \mu \mathrm{M}$ ) (Sigma Aldrich, St. Louis, MO, USA) to determine the optimal dose able to distinguish TMZ sensitivity. Then, 10 $\mathrm{mM}$ of MET (Sigma Aldrich, St. Louis, MO, USA) alone or in combination with $25 \mu \mathrm{M}$ of TMZ was added to the medium once at the beginning of the experiment and cell growth was monitored after 24, 48 and 72 hours (h). Cell viability was evaluated by Trypan blue exclusion test. The effect of MET, TMZ or MET plus TMZ was determined as growth inhibition rate and measured as: $\left[1-\left(\mathrm{C}_{\mathrm{f}} / \mathrm{C}_{0}\right)_{\mathrm{A}} /\left(\mathrm{C}_{\mathrm{f}} / \mathrm{C}_{0}\right)_{\mathrm{V}}\right]^{\star} 100$, where $\mathrm{C}_{\mathrm{f}}$ is the cell number at the point analyzed, $\mathrm{C}_{0}$ is the cell number at the beginning of treatment, $\mathrm{A}$ is the corresponding drug and $\mathrm{V}$ is the vehicle as previously described (12). For L0627 cells, short-term proliferation/survival studies were performed as previously described (32). Apoptosis or necrosis were assessed by Real time-Glo Annexin Apoptosis and Necrosis Assay (Promega Corporation, Madison, Italy).

\section{RNA Extraction and Real-Time PCR}

RNA was extracted using the commercially available illustra RNAspin Mini Isolation Kit (GE Healthcare, Italy), according to manufacturer's instructions. Total RNA was reversetranscribed to cDNA using the High Capacity cDNA Reverse Transcription Kit (Thermo Fisher Scientifics, USA). Real-time PCR was performed in duplicate for each data point by using the Sybr Green technique and the oligonucleotides used were: $\beta$ actin (FRW: TCAAGATCATTGCTCCTCCTG, REV: CCAGA GGCGTACAGGGATAG); bax (FRW: ATG GAC GGG TCC GGG GAG; REV: ATCCAGCCCAACAGCCGC); bad (FRW: C C CA GA T T T GA G C CGAG TG; REV: C C CA T CCCTTCGTCGTCCT); $b c l-2$ (FRW: GATTGTGGCC TTCTTTGAG, REV: CAAACTGAGCAGAGTCTTC); sox2 (FRW: GCACATGAACGGCTGGAGCAACG; REV: TGCT GCGAGTAGGACATGCTGTAGG). Changes in the target mRNA content relative to housekeeping ( $\beta$-actin) were determined with the $\Delta \Delta \mathrm{ct}$ method. Basal level expression of sox 2 gene was expressed as difference between the target mRNA content and the housekeeping $(\beta$-actin $)(\Delta \mathrm{ct})$.

\section{Animal Models and Treatment}

Animal experiments were carried out in compliance with institutional guidelines for the care and the use of experimental animals, which have been authorized by the Italian Ministry of Health ( ${ }^{\circ} 220 / 2016-P R$ and $\left.n^{\circ} 378 / 2019-P R\right)$. Seven to eight weeks old female nude mice (Envigo RMS srl, San Pietro al Natisone, Italy) were housed at constant temperature $\left(23^{\circ} \mathrm{C}\right)$ and relative humidity (40\%) under a regular light/dark cycle, with food and water freely available. The orthotopic tumor models were obtained by the stereotactic injection of $3-5^{\star} 10^{5}$ cells (Gli36 $\Delta$ EGFR-1, Gli36 2 EGFR-1 or L0627) in $2 \mu$ of plain DMEM with a $10 \mu$ Hamilton syringe as previously described (10). After cells injection, mice were monitored every day for body weight and clinical signs of disease (fur, eye, motor impairment) and sacrificed at the appearance of evident signs of illness or at the loss of more than $25 \%$ of the initial body weight. Firstly, we performed a pilot study on mice inoculated with Gli36 $\Delta$ EGFR-1 and with Gli36 $\Delta$ EGFR-2 $(n=6$ per each cell line) to monitor tumor growth with MRI at day 5 after surgery and the sensitivity to TMZ. Based on data obtained on pilot study, we decided to start drug administration 7 days after cells inoculation and perform overall survival studies. Gli36 $\Delta$ EGFR-1 (R-1), Gli36 $\triangle$ EGFR-2 (R-2), and L0627 tumor bearing mice were randomly assigned to 4 groups of treatment, according to the following scheme: Group A ( $\mathrm{n}=5 \mathrm{R}-1 ; \mathrm{n}=13 \mathrm{R}-2$; $\mathrm{n}=10$ L0627) received daily oral administration of Temozolomide (TMZ, 70 $\mathrm{mg} / \mathrm{kg}$ ) in $10 \%$ DMSO, 5 days for a 28 days cycle and repeated with this scheme $(5 / 28)$ until sacrifice of animal; group $B(n=6$ $\mathrm{R}-1 ; \mathrm{n}=5 \mathrm{R}-2 ; \mathrm{n}=5$ L0627) received intra peritoneal (i.p.) administration of Metformin (MET, $250 \mathrm{mg} / \mathrm{kg}$ ) in saline for $5 \mathrm{~d} /$ wk for the entire treatment period; group C $(\mathrm{n}=5 \mathrm{R}-1 ; \mathrm{n}=6$ $\mathrm{R}-2 ; \mathrm{n}=8$ L0627) received the combination of daily oral administration of TMZ $(70 \mathrm{mg} / \mathrm{kg}) 5$ days for a 28 days cycle and i.p. daily administration of MET (250 mg/kg); group D ( $\mathrm{n}=9$ R-1; $\mathrm{n}=9$ R-2; n=10 L0627), as vehicle group, received vehicle administration (10\% DMSO in saline by oral gavage and $100 \%$ saline i.p.). The treatment schedule was decided on the basis of previous studies (12) and of the dose regimen used in clinical practice adapted to mice body surface (Figure S1) (33). The tumor mass presence was confirmed in a subset of animals ( $\mathrm{n}=3$ per treatment group) using MRI as described below. At the onset of signs of severe illness (weight loss $>25 \%$ or hemiplegia), mice were sacrificed under anesthesia and brains collected for histological analyses. Treatment efficacy was evaluated as time to sacrifice indicated as "overall survival" using the KaplanMeier estimator.

\section{Immunohistochemical Analysis}

Mice brains were collected at sacrifice and fixed in $10 \%$ buffered formalin (Sigma-Aldrich) as described (34). After standard histological samples processing, serial 3 um-thick brain sections were cut and stained with hematoxylin and eosin (H\&E) for morphological evaluation or probed with the following primary antibodies: Ki67 (Agilent Technologies, Santa Clara, US.), Sox2 (Cell signaling technologies Leiden, The Netherlands), cleavedCaspase 3 (Cell Signaling Technologies, Palo Alto, CA, USA), and Ibal (Wako pure Chemical Ind. Ltd.). Afterword, slides were 
incubated with Rodent Block $\mathrm{R}$ immunohistochemical reagent (Biocare Medical) before secondary antibody addition. Finally, slides were revealed using $\mathrm{DAB}$ as chromogen and counterstained with hematoxylin. Stained slides were scanned using Aperio digital scanner instrument (Leica Microsystems) and Ibal expression was analyzed using the cytoplasmic algorithm available within ImageScope software (Leica Microsystems) after optimization of cell recognition parameters (12). Briefly, Iba1 quantification was performed by drawing regions of interest of about $0.21 \mathrm{~mm}^{2}$ inside the tumor, in the peripheral inner and outer part of the mass to evaluate intratumor, peritumor or distant active microglia, respectively. Ki67 marker was quantified by drawing the same regions of interest only inside the tumor. The mitotic index in each sample was scored as the average of mitotic cells per $10 \mathrm{High}$ power fields (HPFs; 40X objective) on H\&E slides as previously described (35). If the tumor area was smaller than $10 \mathrm{HPF}$, then the whole tumor tissue was examined for presence of mitotic cells. In supplementary table 1 and 2 mice used in the study are summarized indicating for each animal, treatment condition and day of sacrifice.

\section{In Vivo Imaging}

MRI was performed with a $7 \mathrm{~T}$ small animal magnetic resonance scanner (Bruker, BioSpec 70/30 USR, Paravision 5.1, Germany), equipped with $450 \mathrm{mT} / \mathrm{m}$ gradients (slew-rate: $3400-4500 \mathrm{~T} / \mathrm{m} / \mathrm{s}$; rise-time: $140 \mathrm{~ms}$ ). A phased-array mouse-head coil with four phased-array channels was used as receiver, coupled with a $72 \mathrm{~mm}$ linear-volume coil as transmitter. Mice were anesthetized with isoflurane ( $2 \%$ in oxygen) and positioned prone on a dedicated heated apparatus, to prevent hypothermia. A coronal 2D High Resolution (HR) Rapid Acquisition with Relaxation Enhancement (RARE) T2 and a RARE T1 were acquired. After the injection of $0.2 \mu \mathrm{l} / \mathrm{g}$ of gadobutrol (Gadovist, Bayer Schering Pharma, BerlinWedding, Germany), acquisition of the RARE T1 was repeated. Tumor volume was calculated by manual contour of the postcontrast RARE T1 sequence made by an expert neuroradiologist. Gd-T1-weighted MRI was conducted to verify the presence of lesion before the beginning of treatment and for manual co-registration with PET images performed using PMOD 3.2 software.

PET imaging was performed with $3^{\prime}$-deoxy- $3^{\prime}-\left[{ }^{18} \mathrm{~F}\right]$ fluorothymidine $\left(\left[{ }^{18} \mathrm{~F}\right] \mathrm{FLT}\right)$ and $\left[{ }^{18} \mathrm{~F}\right] \mathrm{VC701}$ to assess proliferation related to TK1 and TSPO receptor expression, respectively. $\left[{ }^{18} \mathrm{~F}\right] \mathrm{FLT}$ and $\left[{ }^{18} \mathrm{~F}\right] \mathrm{VC701}$ uptake was evaluated by PET in distinct groups of Gli36 $\mathrm{EGFR}-1$, Gli36 $\triangle \mathrm{EGFR}-2$ and L0627 mice during control condition $\left(\left[{ }^{18} \mathrm{~F}\right] \mathrm{FLT}: \mathrm{n}=11\right.$ for $\mathrm{R}-1, \mathrm{n}=11$ for R-2 and $\mathrm{n}=9$ for L0627; $\left[{ }^{18} \mathrm{~F}\right] \mathrm{VC701}$ : $\mathrm{n}=9$ for R1,11 for $\mathrm{R}-2$ and $\mathrm{n}=11$ for L0627) and 1 week after the beginning of treatment (vehicle, TMZ, and TMZ plus MET). MET treated animals did not perform imaging. The sample size of each group is indicated in the figures.

Mice were injected via the tail vein with $4.18 \pm 0.28 \mathrm{MBq}$ of $\left[{ }^{18} \mathrm{~F}\right] \mathrm{FLT}$ and $4.79 \pm 0.91 \mathrm{MBq}$ of $\left[{ }^{18} \mathrm{~F}\right]$ VC701. PET acquisitions were performed at $60 \mathrm{~min}\left(\left[{ }^{18} \mathrm{~F}\right] \mathrm{FLT}\right)$ or $120 \mathrm{~min}\left(\left[{ }^{18} \mathrm{~F}\right] \mathrm{VC} 701\right)$ after tracer injection using the YAP-(S)-PET II small animal tomograph (ISE s.r.l., Pisa, Italy) or X-ß-CUBE (Molecubes, Gent, Belgium) as already described $(36,37)$. All the radiopharmaceuticals injected had a radiochemical purity greater than 99\%. PET images were acquired in 3D mode. All the images were co-registered to MRI and quantified with PMOD 3.2 software (Zurich, Switzerland). The quality of coregistration was judged and confirmed by two independent experts in the field (S.V. and I.R.). Two different volumes of interest (VOI) were defined: (i) a control VOI covering the left striatum (volume $7 \mathrm{~mm}^{3}$ ) was drawn on the axial MR images, adjusted on the other imaging planes and then copied on the PET images of each mouse; (ii) a second glioma-covering VOI was drawn in the tumor-affected brain hemisphere and centered on mice lesions. For quantification, Standardized Uptake Value (SUV) was calculated according to the formula: SUV = tumor concentration activity $[\mathrm{MBq} / \mathrm{g}] /($ injected activity $[\mathrm{MBq}] /$ animal weight $[\mathrm{g}]$ ). Maximum tracer uptake in tumor was normalized to the corresponding mean values of uptake of the contralateral control VOI (background) and indicated as tumor to background ratios (T/B ratio). A similar analysis for normal brain parenchyma was performed on normal mice.

\section{Statistical Analysis}

In vitro experiments were repeated three times giving reproducible results. Data are presented as mean values \pm standard deviation (SD) or standard error of the mean (SEM) of three independent experiments. For statistical analysis, non-parametric t-test, oneway analysis of variance (ANOVA), followed by Tukey's multiple comparison test, or two-way ANOVA, followed by Bonferroni's multiple comparison test, were performed using Prism 5 (Graph Pad Software Inc., CA, USA). Log-rank Mantel-Cox test was performed for survival comparison followed by Holm-Sidak method for multiple comparisons correction. Differences were considered statistically significant when $\mathrm{p}<0.05$.

\section{RESULTS}

\section{Combination of Temozolomide and Metformin Reduces Cell Growth and Promotes Early Apoptosis Overcoming TMZ Resistance}

We identified the minimal in vitro dose of TMZ able to reduce Gli36 $\Delta$ EGFR-1 (TMZ-sensitive cells) but not Gli36 $\triangle$ EGFR-2 (TMZ resistant cells) viability. This dose was defined as $25 \mu \mathrm{M}$ for $48 \mathrm{~h}$ (Figure S2). Then, $10 \mathrm{mM}$ of MET alone or in combination with $25 \mu \mathrm{M}$ of TMZ was added to the medium once at the beginning of the experiment and growth of Gli36 $\triangle E$ EFR-1 and Gli36 $\triangle$ EGFR-2 cells was monitored after 24, 48 and 72 hours. The treatment with MET alone induced a significant reduction in cell growth rate only in Gli36 $\triangle E G F R-1$ compared to vehicle ( $\mathrm{p}<0.01$ at $48 \mathrm{~h}$ and $\mathrm{p}<0.001$ at $72 \mathrm{~h}$ ). Furthermore, the growth rate of Gli36 $\triangle$ EGFR-1 was significantly reduced after TMZ treatment at any time ( $p<0.001$ at 24,48 and $72 \mathrm{~h})$. The combination of TMZ and MET (TMZ+MET) displayed an additive effect at $72 \mathrm{~h}$ for Gli36 $\triangle$ EGFR-1 ( $\mathrm{p}<0.01 \mathrm{vs}$ TMZ) and reverted TMZ resistance of Gli36 $\triangle E G F R-2$ cells already after $24 \mathrm{~h}$ of combined treatment $(\mathrm{p}<0.01$ at 24 and $48 \mathrm{~h}$ and $\mathrm{p}<0.05$ at 72h) (Figure 1A), confirming the potential efficacy of MET in reducing cell resistance to TMZ (Figure 1B). The combination of 

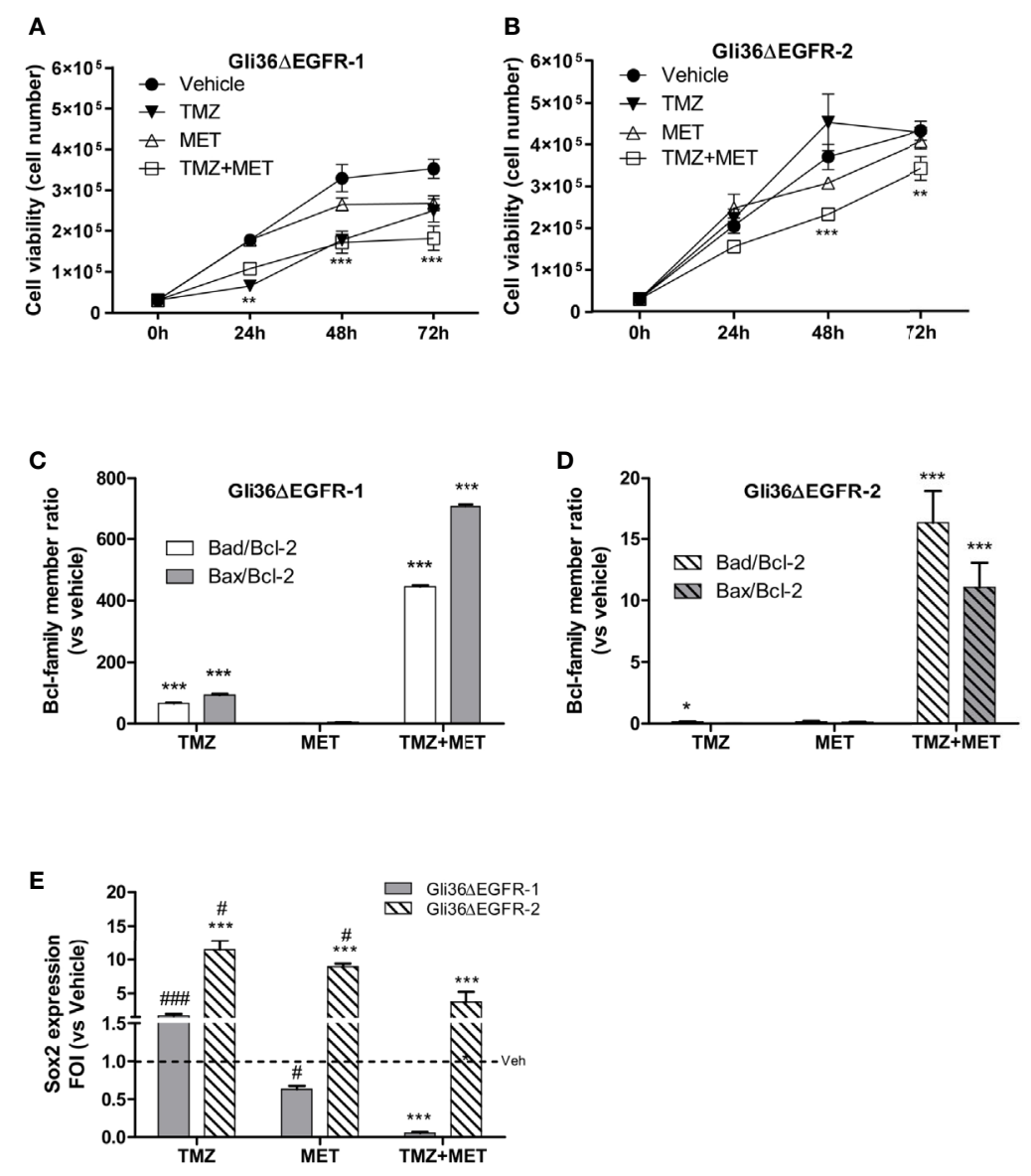

FIGURE 1 | In vitro treatment efficacy. Evaluation of time-response viability of TMZ-sensitive (A) and -resistant (B) cells after vehicle, TMZ, MET or TMZ+MET treatment. Cell viability was assessed by means of a Trypan blue exclusion test and expressed as the number of viable cells after 24,48 or $72 \mathrm{~h}$ of treatment. Twoway ANOVA analysis followed by Bonferroni's multiple comparisons test was performed, ${ }^{\star \star} p<0.01,{ }^{\star \star *} p<0.001$ vs vehicle-treated cells. The induction of proapoptotic (bad and bax) and anti-apoptotic genes (bcl-2) was analyzed by means of real-time PCR in glioma Gli36 $\Delta$ EGFR-1 (C) and Gli36 4 EGFR-2 (D) cells treated with TMZ, MET or the combination and expressed as bax/bcl-2 or bad//bcl-2 ratio. One-way ANOVA analysis followed by Tukey's multiple comparison test was performed, ${ }^{*} p<0.05,{ }^{* *} p<0.01,{ }^{* \star *} p<0.001$. (E), TMZ+MET treatment decreased sox2 expression measured using q-real-time PCR in Gli36 $\triangle E G F R-1$ cells and counteracted its increase in Gli36 $\mathrm{EGFR}-2$ cells. One-way ANOVA analysis followed by Tukey's multiple comparison test was performed, ${ }^{* \star *} \mathrm{p}<0.001$ vs vehicletreated cells; ${ }^{\#} \mathrm{p}<0.05,{ }^{\# \# \#} \mathrm{p}<0.001$ vs TMZ+MET treatment. The molecular data were normalized to $\beta$-actin, and the $\Delta \Delta \mathrm{ct}$ values were expressed as fold of induction (FOI) of the ratio between treated and control cells. Data were expressed as mean values \pm SD of three independent experiments. FOl, fold of induction; MET, metformin; TMZ, temozolomide.

TMZ+MET significantly reduced Gli36 $\triangle$ EGFR-1 cell growth also compared to MET alone ( $<0.01$ at $24 \mathrm{~h}, \mathrm{p}<0.001$ at 48 and $72 \mathrm{~h}$ ). These results were confirmed in a patient-derived Cancer Stem Cell (CSC) line (L0627) that shows features typical of the classic molecular subgroup, such as the overexpression of EGFR gene (30). Also in this case, the combination of TMZ+MET significantly decreased cells survival compared to single therapy at $72 \mathrm{~h}$ ( $\mathrm{p}=0.004$ vs TMZ and $\mathrm{p}=0.012$ vs MET) (Figure S3).

In line with the observed effect on cell viability, the association of TMZ and MET treatments deregulated the balance between pro-survival (bcl2) and pro-apoptotic (bax/bad) Bcl-family members in both Gli36 2 EGFR-1 and Gli36 EEGFR-2 cells (Figures 1C, D) as indicated by the increase of $b a d / b c l-2$ and $\mathrm{bad} / \mathrm{bcl}-2$ ratios. On the contrary, TMZ alone increased $b a d / b c l-2$ mRNA expression only in Gli36 $\mathrm{EGFR}-1$ cells and to a lower extent compared with TMZ+MET treatment. To evaluate the apoptosis induced by treatments, cells were assessed for exposure of Phosphatidylserine (PS) on the outer leaflet of the cell membrane. Supplementary figure 4 showed that only TMZ in Gli36EGFR-1 displayed secondary necrosis with PS translocation to the outer leaflet and loss of membrane integrity. This result suggested that MET and TMZ+MET showed early apoptosis characterized by PS translocation to outer leaflet but no cell membrane disruption (Figure S4).

\section{SOX2 Expression Is Hampered by MET Addition to TMZ}

SOX2 has been reported to play a pivotal role in developing drug resistance of glioma $(11,38)$ and its expression was correlated with the grade of malignancy and favored the maintenance of an 
undifferentiated state of cancer stem cells $(39,40)$. Furthermore, recently its role as glioma stem cell biomarker has been described in association with NANOG and OCT $(41,42)$.

We performed qRT-PCR to investigate if the different treatments could modulate sox 2 expression in TMZ-sensitive and -resistant cells. The basal sox2 mRNA level was similar in Gli36 $\triangle$ EGFR-1 and Gli36 $\mathrm{EGFR}-2$ cells (0.082 and 0.079 respectively). Single-drug treatments did not alter sox 2 expression in Gli36dEGFR-1 cells. In contrast, the combination of the two drugs significantly reduced sox2 $(\mathrm{p}=0.0005)$ levels (Figure 1E). On the contrary, in Gli36 $\mathrm{EGFR}-2$ all treatments led to a dramatic increase of sox 2 expression. However, the increment of sox2 transcript was significantly less pronounced with the combinatorial treatment (Figure 1E). These findings suggest that in TMZ-sensitive cells, the chemotherapeutic perturbed cell viability and partially reduced the determinants of therapy resistance. In this system, the addition of MET was synergistic. On the contrary, TMZ-resistant cells showed an enhanced multidrug resistant phenotype and MET administration was only able to partly reduce TMZ-induced sox2 overexpression. sox 2 is also expressed on L0627 cell (personal communication from R. Galli) but the effects of drugs were not evaluated.

\section{TMZ Plus MET Protocol Increases Survival of Orthotopic GBM Mouse Models}

In a pilot study, after 5 days from cell injection, all animals were positive for intracerebral lesions at MRI (tumor volume: $4.38 \pm$ $1.67 \mathrm{~mm}^{3}$ and $6.12 \pm 2.11 \mathrm{~mm}^{3}$ for Gli36 2 EGFR-1 and Gli36 $\Delta$ EGFR-2 respectively). In the same study we confirmed in vivo the different sensitivity of Gli36 $\triangle \mathrm{EGFR}-1$ and Gli36 $\triangle$ EGFR-2 to TMZ (\% tumor increase after treatment: 22.7 vs 54.9).

Then, we analyzed the efficacy of MET and TMZ treatments alone or in combination in orthotopic mouse models of glioma obtained by Gli36 $\triangle$ EGFR-1 or Gli36 $\triangle$ EGFR-2 cells inoculation. The MET protocol alone did not show any effect on survival of both GBM models. As expected, in the TMZ-sensitive Gli36 $\triangle$ EGFR-1 model, TMZ treatment significantly increased mice overall survival compared to animals treated with vehicle (median survival 63 days vs 17 days, $\mathrm{p}=0.0066$ ). Among TMZtreated mice only one mouse was histologically confirmed as disease-free, while others mice showed tumor relapse detected by MRI or IHC (Figures S5A, B). More interestingly, the combination of TMZ+MET extended the overall survival of Gli36 $\mathrm{EGFR}-1$ mice up to 90 days, when we decided to sacrifice all mice which were histologically confirmed as disease-free (Figure 2A). In mice bearing TMZ-resistant glioma, TMZ alone did not prolong mice survival compared with that of vehicletreated mice (18 days vs 17 days). In this case, TMZ+MET protocol slightly increased median survival time up to 23 days (Figure 2B). EGFR-2 bearing mice regardless of treatment displayed tumor increase at MRI after one week (Figures S5C, D).

When CSC line L0627 was tested in the same settings, orthotopically transplanted mice displayed tumor masses at later times (tumor onset at approximately 40 days) in comparison with Gli36 $\mathrm{EGFR}$ mice. Confirming our previous data, also in the L0627 model, treatment with MET alone did not show any therapeutic efficacy (median survival 54.5 days and 54 days for control and MET, respectively) but, most remarkably, a

\section{A}

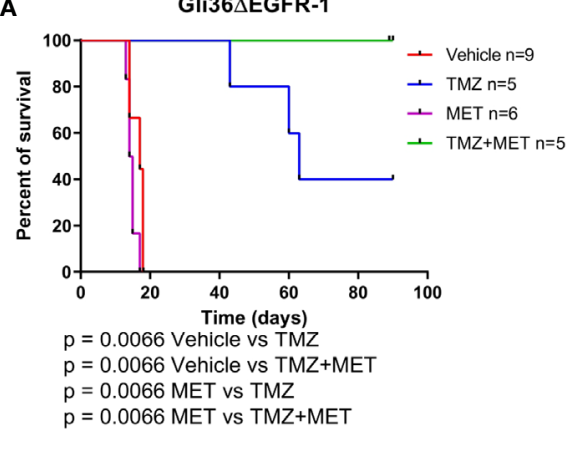

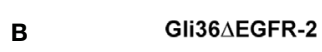

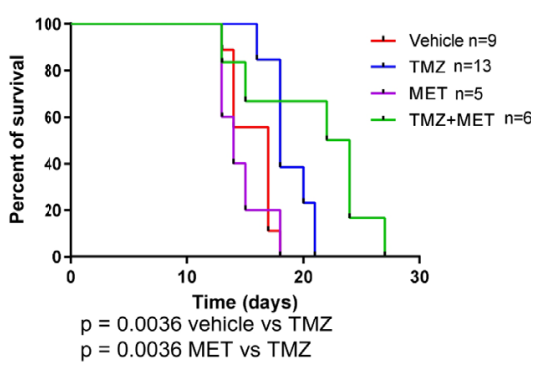

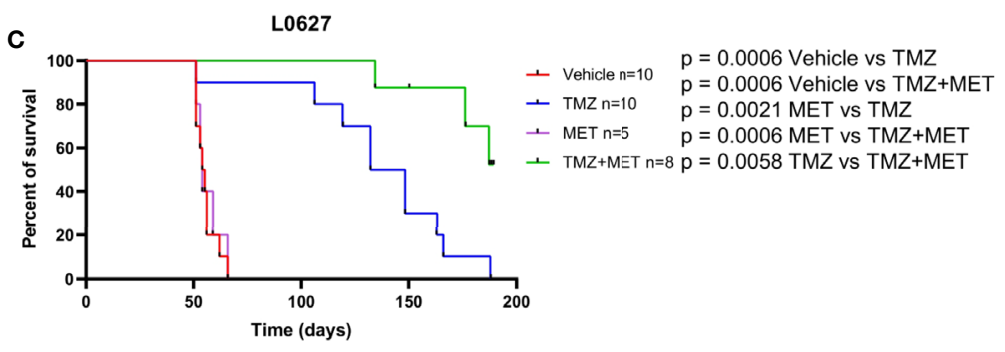

FIGURE 2 | Survival curves of GBM-bearing mice. (A-C), nude mice were injected with Gli36 $\Delta$ EGFR-1, Gli36 $\Delta$ EGFR-2 or L0627 cells. At tumor onset, mice were

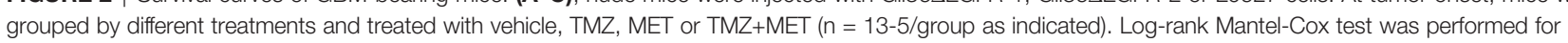
survival comparison followed by Holm-Sidak method for multiple comparisons correction. MET, metformin; TMZ, temozolomide. 
significant increase of survival rate could be detected in animals treated with the MET+TMZ combination in comparison with TMZ alone group ( $\mathrm{p}=0.0058$ ) (Figure 2C). In addition, after an initial response, all mice treated with TMZ showed tumor relapse at the MRI, whereas only 3 out of $8 \mathrm{TMZ}+\mathrm{MET}$ treated animals developed tumor recurrence (Figures S6A, B).

\section{Analysis of Glioma Proliferation Index and Inflammation After Treatment Reveals Superior Anti-Tumor Efficacy of TMZ Plus MET Protocol in Mouse Models of Glioma}

To gain insights into in vivo effects of the different therapeutic protocols, we evaluated glioma proliferation index and inflammation (i.e. microglia/macrophage activation) in mouse brains collected after cancer-related death or sacrifice. Accordingly, Ki67 and Ibal staining were performed and quantified in different brain areas (Figure 3A).

Gli36 $\triangle$ EGFR-1-tumors treated with vehicle showed a mean proliferation index of $58.4 \%$. In the TMZ arm one mouse did not display tumor mass whereas the two tumors processed displayed a proliferation index of $44.4 \%$ and $45.3 \%$. No tumor mass was detected in all samples from the TMZ+MET arm $(n=5)$. For this reason, we also performed STEM121 staining, a marker able to identify human cells in a mouse tissue, which confirmed the absence of human cells. Gli36 $\mathrm{EGFR}-2$ tumors from vehicletreated animals had a mean proliferation index of $40.1 \%$, whereas those from the TMZ or the TMZ plus MET arms showed a mean Ki67 staining of $48.6 \%$ and 40.9\%, respectively (Figures 3B-D, F).

When we looked at microglia/macrophage activation, we did not observe any difference between vehicle and TMZ-treated Gli36 $\Delta$ EGFR-1 tumors (Figure 3E); after treatment with TMZ plus MET no signs of tumor or Ibal markers were visible. In Gli36 $\triangle$ EGFR-2 generated glioma, TMZ+MET significantly reduced infiltration of Ibal positive myeloid cells only at the tumor-brain border compared to TMZ ( $p=0.005$ versus TMZ, Figure 3G). On the other areas data are very heterogeneous.

For the L0627-generated tumors we scored the mitotic count in three high power fields per mouse and tumor cell viability using an antibody toward cleaved-caspase 3, because Ki67 staining was surprisingly absent.

This analysis showed that tumor proliferation was decreased by TMZ or MET individual treatments (20.88 for vehicle, 14.38 for TMZ, 13.90 for MET), but only TMZ marginally increased glioma cells apoptosis $(0.68 \%$ for vehicle, $4.35 \%$ for $\mathrm{TMZ}$ and $2.56 \%$ for MET) (Figures S7A, C). Again, the combinatorial treatment significantly impaired the ability of the glioma cells to in vivo grow, one mouse negative-defined at MRI showed a small tumor mass at sacrifice. In regards of the glioma stem cell markers, we observed a high Nestin staining in all L0627 tumor samples and positive staining for Ibal within and in the peripheral areas of the tumor and in cerebral hemisphere contralateral to tumor implantation (Figure S7B). Interestingly, both MET+TMZtreated analyzed mice (both that with a small tumor mass and that disease-free) displayed Iba1-positive staining only in the cerebral hemisphere contralateral to tumor implantation indicating an activation of macrophages/microglia.

\section{Post-Treatment $\left[{ }^{18} \mathrm{~F}\right] \mathrm{FLT}-\mathrm{PET}$ Uptake Is a Measure of TMZ Responsiveness in the Gli36 $\Delta$ EGFR Model}

We used PET with $\left[{ }^{18} \mathrm{~F}\right] \mathrm{FLT}$ to determine if this tracer could identify responder from non-responder mice after treatment. Gli36 $\triangle$ EGFR-1 and Gli36 $\triangle$ EGFR-2 generated tumors displayed similar $\left[{ }^{18} \mathrm{~F}\right] \mathrm{FLT}$ pre-therapy uptake values, i.e. a SUVmax value of $0.28 \pm 0.06$ and of $0.32 \pm 0.07$, respectively (Figures 4A-D).

Early after therapy start (day 7), $\left[{ }^{18}\right.$ F]FLT uptake increased in all vehicle-treated mice (either carrying a Gli36 $\triangle$ EGFR-1 or Gli36 $\triangle E$ EFR-2 tumors) compared to basal levels ( $p<0.001$; Figure 4C). All treatment protocols significantly reduced the increase of $\left[{ }^{18} \mathrm{~F}\right] \mathrm{FLT}$ uptake both in Gli36 $\mathrm{EGFR}-1$ bearing mice ( $p<0.001$ versus vehicle; Figures 4A, C) and in Gli36 2 EGFR-2 glioma, although to a lower extent (Figures 4B, D).

We successfully identified a cut-off value of $\left[{ }^{18} \mathrm{~F}\right]$ FLT PETSUVmax able to discriminate TMZ-responder (considering both TMZ- and TMZ+MET-treated animals) from therapy-resistant tumors. Appling Receiver Operating Characteristic (ROC) curve to post-treatment $\left[{ }^{18} \mathrm{~F}\right] \mathrm{FLT}$ PET data, we identified a threshold value of SUVmax (corresponding to 0.3295 ) with a sensitivity and specificity of $77.8 \%$ and $100 \%$, respectively, in distinguishing animals response to treatments (Figures 4E, F). Therefore our data suggest that $\left[{ }^{18} \mathrm{~F}\right]$ FLT-PET may be used to monitor treatment responses in glioma and to identify patients as responders or not after treatment. Unfortunately, $\left[{ }^{18} \mathrm{~F}\right] \mathrm{FLT}$ PET was not able to predict response duration.

This study was repeated also in the CSC-transplanted mice. In agreement with the results on Ki67, we didn't observe any significant uptake of $\left[{ }^{18} \mathrm{~F}\right] \mathrm{FLT}$ in L0627 tumors independently from the lesions' dimension or the treatments' protocol, possibly due to the lowly proliferative, highly infiltrative nature of CSCs and to the maintenance of Blood Brain Barrier (BBB) integrity in this specific GBM model (43).

\section{The Uptake of the Inflammatory-Related $\left[{ }^{18}\right.$ F]VC701-PET Probe Was Reduced by TMZ Plus MET Treatment in Gli36 $\triangle E$ GFR-1 Tumors and Negatively Associated With Treatment Response}

After 7 days from the beginning of treatment, vehicle- or TMZtreated Gli36 $\triangle$ EGFR-1 glioma mice displayed the same uptake of $\left[{ }^{18} \mathrm{~F}\right] \mathrm{VC701}$ of the pre-treatment mice. On the contrary, the TMZ plus MET protocol in Gli36 $\mathrm{EGFR}-1$ glioma led to a significant decrease of $\left[{ }^{18} \mathrm{~F}\right]$ VC701 uptake $(0.15 \pm 0.07$ SUVmax $)$ not only in comparison with mice treated with vehicle $(0.35 \pm 0.07, \mathrm{p}<0.01)$ or TMZ alone $(0.31 \pm 0.08, \mathrm{p}<0.01)$ but also in comparison with pretreatment uptake $(0.29 \pm 0.07, \mathrm{p}<0.05)$ (Figures 4B, 5A). Moreover, in this model we observed an increased uptake of $\left[{ }^{18} \mathrm{~F}\right]$ VC701 in brain regions contralateral to tumor mass regardless of treatment conditions compared to that of healthy brains $(\mathrm{p}<0.05)$, except animals treated with TMZ plus MET (SUVmax values: pretreatment $=0.27 \pm 0.05$; vehicle $=0.28 \pm 0.06$; $\mathrm{TMZ}=0.29 \pm 0.08$; MET plus TMZ $=0.14 \pm 0.07$ and healthy brain $=0.16 \pm 0.05$ )

(Figures S8A, B). In Gli36 $\triangle E$ EFR-1 samples, the expression of Iba1 
A
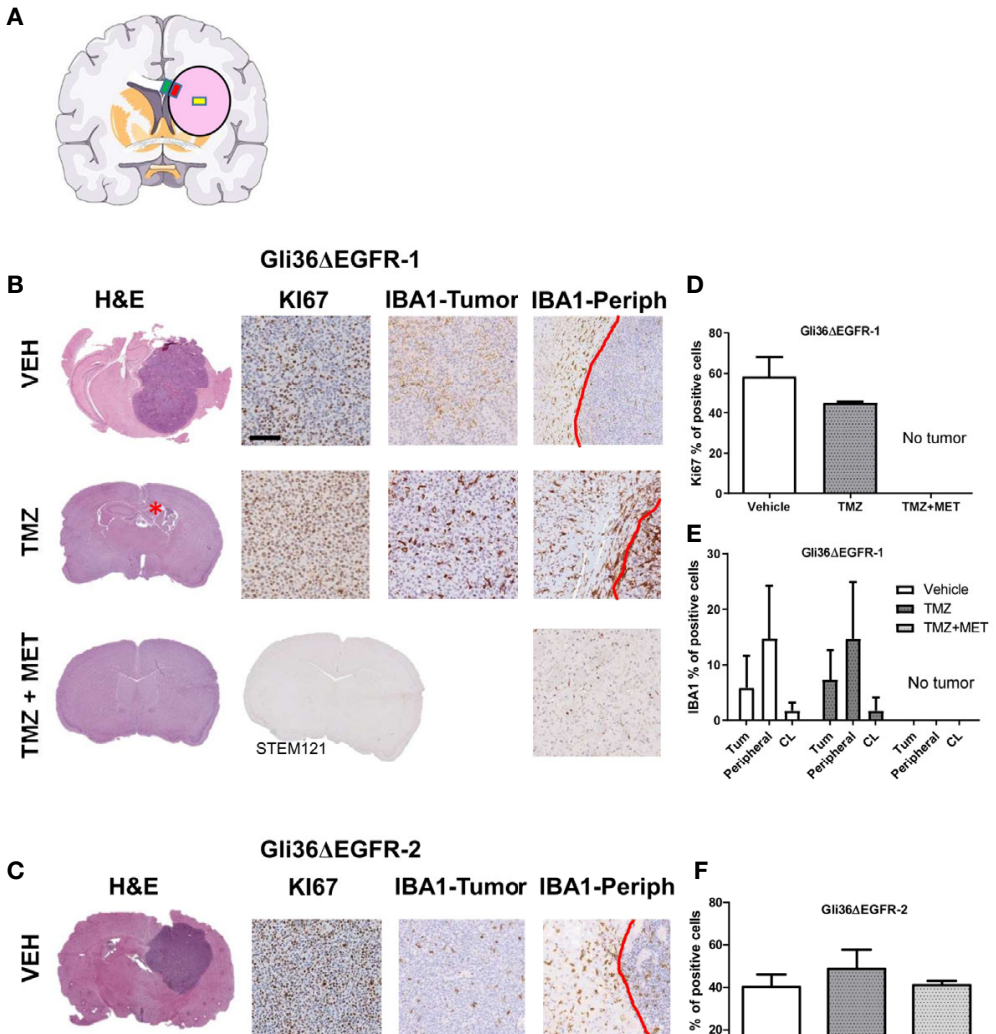

Gli36 $\triangle E G F R-2$

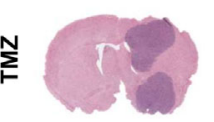

KI67
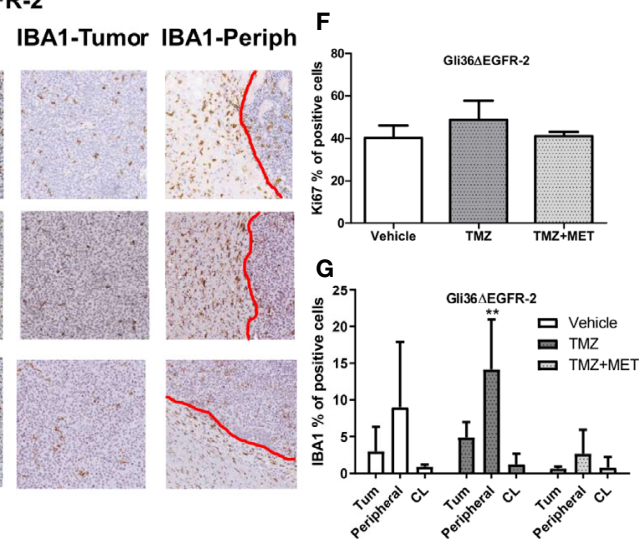

FIGURE 3 | Evaluation of KI67 and IBA1 in GBM mouse samples at the end of survival study. (A), schematic representation of the brain areas sampled for Iba1 quantification. The pink circle indicates the tumors, the yellow square indicates the Regions of Interest (ROI) placed on the tumor, the red one that placed on the inner border, and the green one that placed on the outer border. (B, C), images of representative hematoxylin and eosin (H\&E), Ki67 and Iba1 staining for intracranial Gli36 $\triangle E G F R-1$ and Gli36 $\triangle E G F R-2$ tumors from mice treated with vehicle (VEH, $n=5$ for R1, $n=3$ for R2), TMZ ( $n=2$ for R1, $n=4$ for R2) and TMZ+MET ( $n=5$ for R1 and $n=3$ for R2) euthanized at the end of survival study. Gli36 $\triangle E G F R-1$ TMZ+MET tumors were stained also with STEM121. (D, F), quantification of the ki67 marker expressed as the percentage of positive cells. One-way ANOVA analysis followed by Tukey's multiple comparison test. (E, G), quantification of IBA1 marker expressed as percentage of positive cells in the inner part of the tumor (Tum), in tumor-brain border (Peripheral) and in the brain region contralateral to the tumor (CL). Two-way ANOVA analysis followed by Bonferroni's multiple comparison test, ${ }^{* *} \mathrm{p}=0.0050 \mathrm{TMZ}+\mathrm{MET}$ vs TMZ.

staining was very heterogeneous in the cerebral hemisphere contralateral to tumor implantation (Figure 3B) and it was correlated to expression of Iba1 in outer border of tumor (R2 = $0.68, \mathrm{p}=0.02$ ). No expression of Ki67 proliferation marker was detected in these areas. The increased uptake observed in the normal brain parenchyma of tumor bearing animals suggests that Gli36 $\mathrm{EGFR}-1$ tumors enhance the inflammation status of the whole brain, an effect that is blocked by the combination of MET to TMZ. Interestingly, within the TMZ-responder Gli36 $\triangle E G F R-1$ group (TMZ- and TMZ+MET-treated animals) identified with $\left[{ }^{18} \mathrm{~F}\right]$ FLT PET we observed a significant inverse correlation between $\left[{ }^{18} \mathrm{~F}\right] \mathrm{VC701}$ uptake and survival $(\mathrm{r}=-0.8674, \mathrm{p}=$ 0.0252 ) with long-term survived mice (disease-free mice at 90 days independently by type of treatment) displaying lower $\left[{ }^{18} \mathrm{~F}\right]$ VC701 uptake values (Figures 5C, D).

On the contrary, in Gli36 $\triangle$ EGFR-2 tumor bearing mice, $\left[{ }^{18} \mathrm{~F}\right]$ VC701 uptake was lower in comparison with Gli36 2 EGFR-1 bearing mice and not affected by treatment (Figure 5B and Figures S8A, B).

Also in this case, L0627 tumors displayed a different behavior. We observed a slight but significant decrease of $\left[{ }^{18} \mathrm{~F}\right] \mathrm{VC} 701$ uptake in the tumor after 7 days of treatment with TMZ plus 
A

Gli36 $\triangle$ EGFR-1
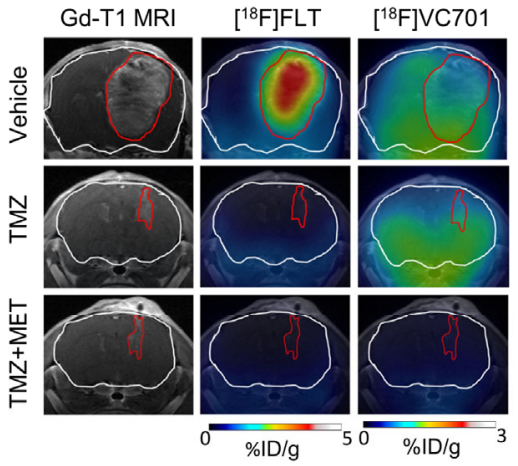

B
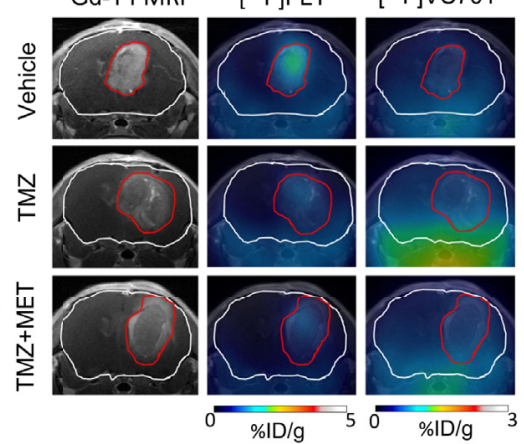

C

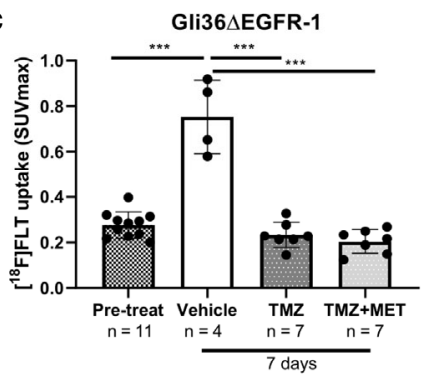

E

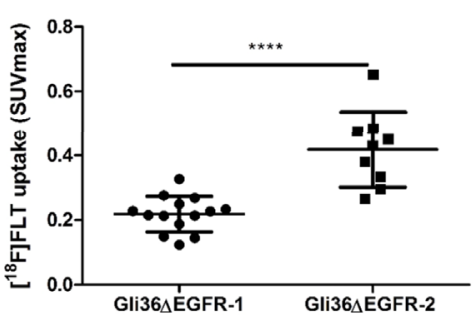

D

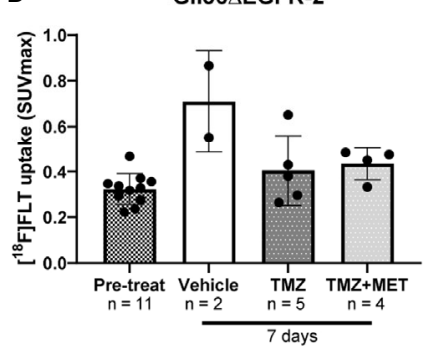

F

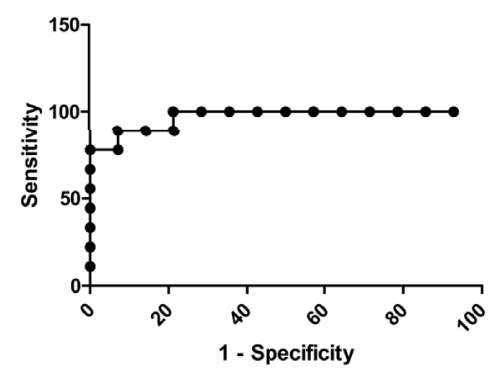

FIGURE 4 | [ $\left.{ }^{18} \mathrm{~F}\right] \mathrm{FLT}$ uptake distinguished between TMZ-sensitive and -resistant tumors. Representative Gadolinium-T1w MRI images and their fusion with PET images for $\left[{ }^{18} \mathrm{FFLT}\right.$ and $\left[{ }^{18} \mathrm{~F}\right.$ VC701 of Gli36 $\mathrm{EEGFR}-1$ (A) and Gli36 $\Delta$ EGFR-2 tumor-bearing mice (B) after 7 days of vehicle, TMZ, and TMZ+MET treatment. The white line indicates the brain and the red one indicates the tumor area depicted by MRI. ${ }^{18} \mathrm{~F}$ FLT uptake expressed as SUVmax in Gli36 $\Delta$ EGFR-1 (C) and in Gli36 $\Delta$ EGFR-2 (D) tumor-bearing mice before and after 7 days of treatment. One-way ANOVA analysis followed by Tukey's multiple comparison test was performed, ${ }^{* * *} \mathrm{p}<0.001$. In the bottom part of both graphs, the sample size for each group is indicated. (E), After 7 days of treatment with TMZ or TMZ+MET, mice with TMZ-resistant tumors showed a significant increase of $\left[{ }^{18} \mathrm{~F}\right] \mathrm{FLT}$ uptake compared to TMZ-sensitive ones. Unpaired t-test analysis was performed, ${ }^{\star \star \star \star} \mathrm{p}<0.0001$. (F), ROC analysis of $\left[{ }^{18} \mathrm{~F}\right.$ FLT SUVmax for prediction of different response to TMZ therapy. Optimal cut-off point was defined for [ $\left.{ }^{18} \mathrm{~F}\right] \mathrm{FLT}$ as $0.3295(77.8 \%$ sensitivity; $100 \%$ specificity).

MET in comparison with vehicle animals and no significant modifications in the contralateral part of the brain both in untreated and treated mice (Figure S9).

\section{DISCUSSION}

We and others reported that MET exerts a synergic action with TMZ and it is able to revert TMZ resistance in GBM models (11, $12,44,45)$. In this study, we further evaluated the effect of MET used in combination with TMZ on two genetically homogeneous GBM models either sensitive (Gli36 $\mathrm{EGFR}-1)$ or resistant (Gli36 $\triangle$ EGFR-2) to TMZ and carrying EGFRvIII mutation, representative of the classical molecular subgroup. Afterwards, we took advantage of a patient-derived Cancer Stem Cell (CSC) line (L0627) that shows features typical of the classic molecular phenotype such as the overexpression of EGFR gene to validate the results also in a preclinical model closer to clinic.

MET increases the effect of TMZ on tumor growth arrest and apoptotic markers. However, the effect of MET add-on was higher for the TMZ sensitive cell line. The differences observed may be related to the significant reduction of sox2 levels induced by TMZ plus MET only on Gli36 $\triangle E$ EFR-1. sox2 is a typical marker of poor differentiated cells that have been associated with CSC and tumor aggressiveness whose levels are regulated by several pathways including EGFR $(39,41,42)$. Indeed, high levels of sox 2 are described in presence of EGFRvIII mutation as is the case of our cell lines. Moreover, modulation of sox2 is able to 
A

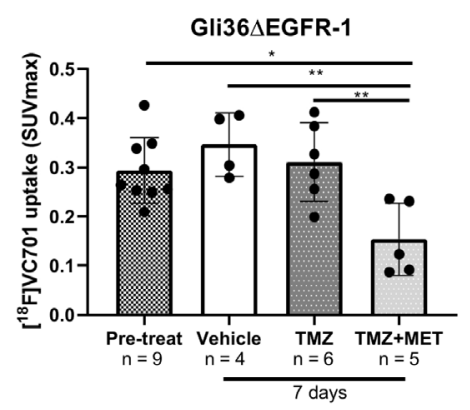

C

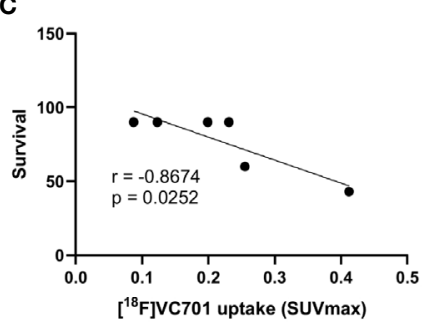

B

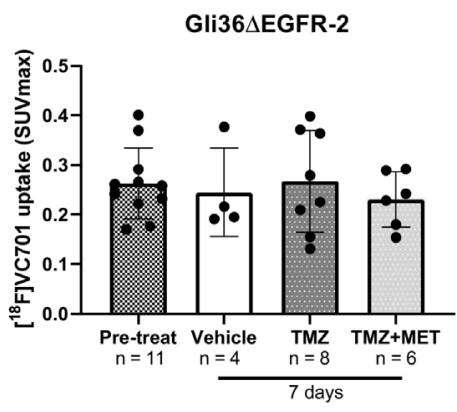

D

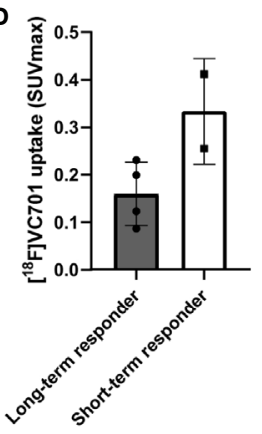

FIGURE $5 \mid\left[^{18} \mathrm{~F}\right]$ VC701 uptake in GBM mouse models. (A), $\left[{ }^{18} \mathrm{~F}\right]$ VC701 uptake expressed as SUVmax in Gli36 $\triangle \mathrm{EGFR}-1$ tumor-bearing mice decreased after 7 days treatment with TMZ+MET. One-way ANOVA analysis followed by Tukey's multiple comparison test. ${ }^{*} \mathrm{p}<0.05,{ }^{* *} \mathrm{p}<0.01$. (B), No changes of $\left[{ }^{18} \mathrm{FVC701}\right.$ uptake was observed in Gli36 $E$ EGFR-2 tumor-bearing mice. (C) Correlation curve between $\left[{ }^{18} \mathrm{~F}\right] \mathrm{VC} 701$ uptake and survival in TMZ-responder Gli36 $\Delta E G F R-1$ mice $(r=$ -0.8674, $p=0.0252$ ). (D) Graph of $\left[{ }^{18} \mathrm{~F}\right] \mathrm{VC} 701$ uptake expressed as SUVmax of Gli36 $\mathrm{EGFR}-1$ long-term (disease-free mice at 90 days independently by type of treatment) and short-term survived mice. Unpaired t-test analysis was performed.

oppositely increase or reduce TMZ sensitivity $(38,46)$. In Gli36 $\triangle E$ EFR-1, MET and particularly MET plus TMZ showed a decrease of sox 2 when compared to TMZ given alone. This effect was not observed in Gli36 $\mathrm{EGFR}-2$ where a marked increase in sox 2 was detected with all treatments although its increase was lower when MET was associated with TMZ. An effect of MET on sox2 concerning cell viability and animal survival on TMZ resistant cell models was previously described by Yang et al. (11). However, in their case cells were repeatedly exposed to MET prior to animal inoculation.

During in vivo studies, TMZ administration increased animal survival only in the sensitive cell line model. As previously observed on U251 and T98 cells, MET given alone in doses closed to those used for diabetes treatment failed to increase animal survival independently of cell line model (12). In agreement with in vitro results, MET potentiates the effect of TMZ. However, also in this case, the effect was higher in animals carrying Gli36 $\triangle$ EGFR-1 cells. Overall survival studies were supported by post mortem IHC analysis. Gli36 $\triangle$ EGFR-2-bearing mice revealed high levels of Ki67 independently on treatment schedule, confirming that in the case of EGFR mutated TMZ-resistant cells, MET is not able to efficiently counteract TMZ resistance. Treatment modulation on cell proliferation was confirmed also by PET study with $\left[{ }^{18} \mathrm{~F}\right] \mathrm{FLT}$. Indeed, $\left[{ }^{18} \mathrm{~F}\right] \mathrm{FLT}$ uptake was reduced at early times by TMZ or TMZ plus MET treatment in mice bearing Gli36 $\mathrm{EGFR}-1$ and post-treatment $\left[{ }^{18} \mathrm{~F}\right] \mathrm{FLT}$ SUV values were able to non-invasively quantify the extent of response in responder from non-responder subjects. Unfortunately, using $\left[{ }^{18} \mathrm{~F}\right] \mathrm{FLT}$ PET was not possible to differentiate long-term responder mice from short-term responder mice. Results on in vitro and in vivo cell growth and animal survival were confirmed on L0627, indicating that MET is able to increase TMZ effect also on CSC lines and CSC-derived xenografts. Interestingly, both in L0627 and Gli36 2 EGFR-1 model, longitudinal MRI studies showed that TMZ administration reduced or eliminated lesions after one week (Figures S5A, B and S6). However, at variable times after the beginning of treatment, we observed lesion relapse in all animals treated with TMZ alone. This effect was absent or definitely lower when MET was added to TMZ suggesting that the combination treatment is able to prevent some mechanisms associated with TMZ-induced tumor resistance. A low level of $\left[{ }^{18} \mathrm{~F}\right] \mathrm{FLT}$ uptake was observed in $\mathrm{L} 0627$ mice precluding the use of $\left[{ }^{18} \mathrm{~F}\right]$ FLT-PET as early predictor. Kinetics modelling evidences suggest that in brain tumors, $\left[{ }^{18} \mathrm{~F}\right] \mathrm{FLT}$ uptake is tightly dependent on factors related to influx (BBB integrity, permeability and transport expression) and not only to thymidine kinase-1 activity (47). The low proliferative, highly infiltrative nature of CSCs in general and of these cells in particular and the maintenance of BBB integrity in this L0267 GBM model could partly contribute to the lower uptake of $\left[{ }^{18} \mathrm{~F}\right] \mathrm{FLT}$ observed (43).

Several evidences indicate that, in glioma, the inflammatory component increases in parallel with tumor aggressiveness and grade (48). Furthermore, glioma cells are able to recruit glioma 
associated peripheral monocyte/macrophage and activated microglia (GAMMs) favoring a tumorigenic phenotype $(49,50)$ which plays a central role in drug resistance and treatment failures. Recent findings suggest that MET is able to target the inflammatory component associated with the cancer microenvironment $(22,51)$. In both Gli36 $\mathrm{EGFR}$ models, we found positive Ibal staining particularly in TMZ sensitive lesions, distributed with a decreasing gradient from outer border of the tumor to the inner regions. In Gli36 $\triangle$ EGFR-1 and in L0627models, sparse Iba1 signal was also present in the brain parenchyma contralateral to tumor. This data is in agreement with what recently reported by Foray et al. (52). The authors found in Gli36 $\triangle E G F R-1$ model an increased number of peri-tumoral GAMMs and astrocytes expressing TSPO and an augmented expression of M2 phenotype myeloid markers in TMZ-treated animals. Gabrusiewicz et al., in a GL261 glioma mice model, described the presence of microglia/macrophage infiltrating cells already at 5 days after cell implantation also in the contralateral, non-tumors containing hemispheres (53) and Guardia Clausi et al. observed modifications of inflammatory cells not only in the tumor but also in the cerebral hemisphere contralateral to tumor implantation after therapy (54). In some types of cancer, MET is able to switch macrophage polarization versus M1 phenotype and this effect might participate to the capability of MET to counteract TMZ recurrences (55). Our results of TSPO PET studies performed at early time showed that $\left[{ }^{18} \mathrm{~F}\right] \mathrm{VC} 701$ was taken up particularly by Gli36 $\mathrm{EGFR}-1$ GBM. High radioactivity uptake was observed not only in tumors but also in extra tumor regions in comparison with normal healthy mice. In addition, administration of MET plus TMZ was the only treatment able to reduce the $\left[{ }^{18} \mathrm{~F}\right] \mathrm{VC} 701$ signal in brain regions surrounding the tumors as well as in normal brain parenchyma and extra-cerebral tissues of Gli36 $\triangle \mathrm{EGFR}-1$ but not in that of Gli36 $\triangle$ EGFR-2 nor L0627 suggesting that the inflammatory signal is cell line-specific as well as their treatmentinduced modulation. On the contrary to $\left[{ }^{18} \mathrm{~F}\right] \mathrm{FLT}$, in TMZresponder Gli36 $\triangle$ EGFR-1 group (TMZ- and TMZ+MET-treated animals) we observed a significant inverse correlation between $\left[{ }^{18} \mathrm{~F}\right]$ VC701 uptake and survival. Long-term survived mice (disease-free mice at 90 days independently by type of treatment) displayed lower $\left[{ }^{18} \mathrm{~F}\right]$ VC701 uptake values. Although $\left[{ }^{18} \mathrm{~F}\right] \mathrm{FLT}$ signal aligns with therapy response monitored with MRI, $\left[{ }^{18} \mathrm{~F}\right] \mathrm{FLT}$ gives information about a biological feature of the tumor and $\left[{ }^{18} \mathrm{~F}\right]$ FLT uptake area not necessarily overlapped with tumor volume by identifying specific areas with higher cell proliferation (56). Moreover, the combination of two radiotracers could allow to obtain complementary information to better monitor therapy response.

Firstly used to image glioma by Junck et al. (57), post-surgery studies showed that TSPO receptors are localized on tumors cells particularly in high grade glioma $(58,59)$. Moreover, using IHC, $\mathrm{Su}$ et al. demonstrated that a relevant percentage of glioma associated macrophage is negative for TSPO (60). These findings were reported also by preclinical studies on orthotopic mouse models (including the model described in the present study) showing that both tumors and microglia/macrophage components contribute to TSPO related PET signal (56). The TSPO role in GBM tumors cells is not clear. Fu et al. showed that absence rather than the increase of TSPO favors an aggressive phenotype of tumors with increased proliferation rate, glycolytic metabolism and poor outcome (61). A better knowledge of the role and the expression of TSPO is surely fundamental to use the TSPO-imaging in clinical practice but this method can be useful to monitor reactive cell infiltration.

Overall, our data confirm the lack of effect of MET given alone reported in our previous work. In addition, we showed that the association between MET and TMZ increases the treatment efficacy in mouse GBM models with EGFR-mutated or -amplified not previously exposed to TMZ. Finally, as indicated by longitudinal studies with MRI, MET association reduces the rate of recurrence during TMZ treatment. However, in a recent retrospective cohort study, Seliger et al. observed that the use of metformin was associated with better overall and progression-free survival of patients with WHO grade III associated with IDH1 mutations (62). For this reason, our results need to be confirmed in other GBM models particularly associating radiotherapy to pharmacological treatment. In our study, PET imaging with $\left[{ }^{18} \mathrm{~F}\right] \mathrm{FLT}$ suggests that the reduction of cell proliferation represents a common mechanism of response for TMZ whereas only TMZ plus MET was able to decrease the in vivo binding of $\left[{ }^{18} \mathrm{~F}\right] \mathrm{VC} 701$ to TSPO receptors, an effect that was associated with animals survival. These results suggest that TSPO-ligand may be used as a complementary molecular imaging marker to predict tumor microenvironment related treatment effects.

\section{DATA AVAILABILITY STATEMENT}

The raw data supporting the conclusions of this article will be made available by the authors, without undue reservation.

\section{ETHICS STATEMENT}

The animal study was reviewed and approved by Ethics Committee of IRCCS San Raffaele Scientific Institute of Milan and Italian Ministry of Health ( ${ }^{\circ} 220 / 2016-P R$ and $n^{\circ} 378 /$ 2019-PR).

\section{AUTHOR CONTRIBUTIONS}

SV and RM designed the study. SV, ALD, ST, BZ, ED, AC, LP, TV, VV, PR, and VP developed the methodology. SV, ALD, IR, CM, VP, and VV performed research and data analysis. SV, ALD, AJ, VP, RG, LO, VV, and RM drafted the manuscript. All authors contributed to the article and approved the submitted version.

\section{FUNDING}

This research was funded by Associazione Italiana Ricerca sul Cancro (AIRC) IG 2014 grant n. 15771 and IG 2018 grant n. 21635 (RM) and by grants from the SysBioNet project, a MIUR initiative for the Italian Roadmap of European Strategy Forum on Research Infrastructures (ESFRI). The Italian Ministry for Education and Research (MIUR) is gratefully acknowledged for 
yearly FOE funding to the Euro-BioImaging Multi-Modal Molecular Imaging Italian Node (MMMI).

\section{ACKNOWLEDGMENTS}

The authors thank P. Simonelli, Dr. E. Turolla, S. Stucchi and Dr. S. Battaglia of University of Milano-Bicocca for their technical support in FLT synthesis and animal handling, T. Canu of CIS-

\section{REFERENCES}

1. Stupp R, Mason WP, van den Bent MJ, Weller M, Fisher B, Taphoorn MJB, et al. Radiotherapy Plus Concomitant and Adjuvant Temozolomide for Glioblastoma. N Engl J Med (2005) 352:987-96. doi: 10.1056/NEJMoa043330

2. Gaspar N, Marshall L, Perryman L, Bax DA, Little SE, Viana-Pereira M, et al. Mgmt-Independent Temozolomide Resistance in Pediatric Glioblastoma Cells Associated With a PI3-Kinase-Mediated Hox/Stem Cell Gene Signature. Cancer Res (2010) 70:9243-52. doi: 10.1158/0008-5472.CAN-10-1250

3. Herrlinger U, Tzaridis T, Mack F, Steinbach JP, Schlegel U, Sabel M, et al. Lomustine-Temozolomide Combination Therapy Versus Standard Temozolomide Therapy in Patients With Newly Diagnosed Glioblastoma With Methylated MGMT Promoter (CeTeG/NOA-09): A Randomised, Open-Label, Phase 3 Trial. Lancet (2019) 393:678-88. doi: 10.1016/S01406736(18)31791-4

4. Liang J, Cao R, Zhang Y, Xia Y, Zheng Y, Li X, et al. PKM2 Dephosphorylation by Cdc25A Promotes the Warburg Effect and Tumorigenesis. Nat Commun (2016) 7:12431. doi: 10.1038/ncomms12431

5. Caniglia JL, Jalasutram A, Asuthkar S, Sahagun J, Park S, Ravindra A, et al. Beyond Glucose: Alternative Sources of Energy in Glioblastoma. Theranostics (2021) 11:2048-57. doi: 10.7150/thno.53506

6. Mazurek M, Litak J, Kamieniak P, Kulesza B, Jonak K, Baj J, et al. Metformin as Potential Therapy for High-Grade Glioma. Cancers (2020) 12:210. doi: $10.3390 /$ cancers 12010210

7. Bi J, Chowdhry S, Wu S, Zhang W, Masui K, Mischel PS. Altered Cellular Metabolism in Gliomas - an Emerging Landscape of Actionable CoDependency Targets. Nat Rev Cancer (2020) 20:57-70. doi: 10.1038/ s41568-019-0226-5

8. Brown SL, Kolozsvary A, Isrow DM, Al Feghali K, Lapanowski K, Jenrow KA, et al. A Novel Mechanism of High Dose Radiation Sensitization by Metformin. Front Oncol (2019) 9:247. doi: 10.3389/fonc.2019.00247

9. Calvo Tardón M, Marinari E, Migliorini D, Bes V, Tankov S, Charrier E, et al. An Experimentally Defined Hypoxia Gene Signature in Glioblastoma and Its Modulation by Metformin. Biology (2020) 9:264. doi: 10.3390/biology9090264

10. Sesen J, Dahan P, Scotland SJ, Saland E, Dang V-T, Lemarié A, et al. Metformin Inhibits Growth of Human Glioblastoma Cells and Enhances Therapeutic Response. PloS One (2015) 10:e0123721. doi: 10.1371/ journal.pone.0123721

11. Yang SH, Li S, Lu G, Xue H, Kim DH, Zhu J-J, et al. Metformin Treatment Reduces Temozolomide Resistance of Glioblastoma Cells. Oncotarget (2016) 7:78787-803. doi: 10.18632/oncotarget.12859

12. Valtorta S, Lo Dico A, Raccagni I, Gaglio D, Belloli S, Politi LS, et al. Metformin and Temozolomide, a Synergic Option to Overcome Resistance in Glioblastoma Multiforme Models. Oncotarget (2017) 8:113090-104. doi: 10.18632/oncotarget.23028

13. Lo Dico A, Valtorta S, Ottobrini L, Moresco RM. Role of Metformin and AKT Axis Modulation in the Reversion of Hypoxia Induced TMZ-Resistance in Glioma Cells. Front Oncol (2019) 9:463. doi: 10.3389/fonc.2019.00463

14. Verhaak RGW, Hoadley KA, Purdom E, Wang V, Qi Y, Wilkerson MD, et al. Integrated Genomic Analysis Identifies Clinically Relevant Subtypes of Glioblastoma Characterized by Abnormalities in PDGFRA, Idh1, EGFR, and NF1. Cancer Cell (2010) 17:98-110. doi: 10.1016/j.ccr.2009.12.020

15. Brennan CW, Verhaak RGW, McKenna A, Campos B, Noushmehr H, Salama SR, et al. The Somatic Genomic Landscape of Glioblastoma. Cell (2013) 155:462-77. doi: 10.1016/j.cell.2013.09.034
San Raffaele Scientific Institute for MRI acquisition and Prof. A. Cappelli of University of Siena for VC701 chemical synthesis.

\section{SUPPLEMENTARY MATERIAL}

The Supplementary Material for this article can be found online at: https://www.frontiersin.org/articles/10.3389/fonc.2021. 664149/full\#supplementary-material

16. Felsberg J, Hentschel B, Kaulich K, Gramatzki D, Zacher A, Malzkorn B, et al. Epidermal Growth Factor Receptor Variant III (Egfrviii) Positivity in EGFR -Amplified Glioblastomas: Prognostic Role and Comparison Between Primary and Recurrent Tumors. Clin Cancer Res (2017) 23:6846-55. doi: 10.1158/ 1078-0432.CCR-17-0890

17. Guo G, Narayan R, Horton L, Patel T, Habib A. The Role of EGFR-Met Interactions in the Pathogenesis of Glioblastoma and Resistance to Treatment. CCDT (2017) 17:297-302. doi: 10.2174/1568009616666161215162515

18. Ciaglia E, Abate M, Laezza C, Pisanti S, Vitale M, Seneca V, et al. Antiglioma Effects of N6-isopentenyladenosine, an Endogenous Isoprenoid End Product, Through the Downregulation of Epidermal Growth Factor Receptor: Antiglioma Action of IPA by AMPK-dependent EGFR Loss. Int J Cancer (2017) 140:959-72. doi: 10.1002/ijc.30505

19. Yeung Y, McDonald K, Grewal T, Munoz L. Interleukins in Glioblastoma Pathophysiology: Implications for Therapy: Targeting Ils in Glioblastoma. $\mathrm{Br} \mathrm{J}$ Pharmacol (2013) 168:591-606. doi: 10.1111/bph.12008

20. Gurgis FMS, Yeung YT, Tang MXM, Heng B, Buckland M, Ammit AJ, et al. The P38-MK2-HuR Pathway Potentiates Egfrviii-IL-1 $\beta$-Driven IL-6 Secretion in Glioblastoma Cells. Oncogene (2015) 34:2934-42. doi: 10.1038/ onc. 2014.225

21. Wu A, Maxwell R, Xia Y, Cardarelli P, Oyasu M, Belcaid Z, et al. Combination anti-CXCR4 and anti-PD-1 Immunotherapy Provides Survival Benefit in Glioblastoma Through Immune Cell Modulation of Tumor Microenvironment. J Neurooncol (2019) 143:241-9. doi: 10.1007/s11060-019-03172-5

22. Chiang C-F, Chao T-T, Su Y-F, Hsu C-C, Chien C-Y, Chiu K-C, et al. Metformin-Treated Cancer Cells Modulate Macrophage Polarization Through AMPK-NF- Kb Signaling. Oncotarget (2017) 8:20706-18. doi: 10.18632 /oncotarget.14982

23. Zinnhardt B, Roncaroli F, Foray C, Agushi E, Osrah B, Hugon G, et al. Imaging of the Glioma Microenvironment by TSPO Pet. Eur J Nucl Med Mol Imaging (2021). doi: 10.1007/s00259-021-05276-5

24. Corroyer-Dulmont A, Pérès EA, Petit E, Guillamo J-S, Varoqueaux N, Roussel $\mathrm{S}$, et al. Detection of Glioblastoma Response to Temozolomide Combined With Bevacizumab Based on $\mu \mathrm{mri}$ and $\mu$ pet Imaging Reveals [18F]-Fluoro-LThymidine as an Early and Robust Predictive Marker for Treatment Efficacy. Neuro-Oncology (2013) 15:41-56. doi: 10.1093/neuonc/nos260

25. Pigeon H, Pérès EA, Truillet C, Jego B, Boumezbeur F, Caillé F, et al. Tspo-PET and Diffusion-Weighted MRI for Imaging a Mouse Model of Infiltrative Human Glioma. Neuro-Oncology (2019) 21:755-64. doi: 10.1093/neuonc/noz029

26. Nishikawa R, Ji XD, Harmon RC, Lazar CS, Gill GN, Cavenee WK, et al. A Mutant Epidermal Growth Factor Receptor Common in Human Glioma Confers Enhanced Tumorigenicity. Proc Natl Acad Sci (1994) 91:7727-31. doi: 10.1073/pnas.91.16.7727

27. Abe T, Wakimoto H, Bookstein R, Maneval DC, Chiocca EA, Basilion JP. IntraArterial Delivery of p53-containing Adenoviral Vector Into Experimental Brain Tumors. Cancer Gene Ther (2002) 9:228-35. doi: 10.1038/sj.cgt.7700437

28. Viel T, Schelhaas S, Wagner S, Wachsmuth L, Schwegmann K, Kuhlmann M, et al. Early Assessment of the Efficacy of Temozolomide Chemotherapy in Experimental Glioblastoma Using [18F]FLT-PET Imaging. PloS One (2013) 8: e67911. doi: 10.1371/journal.pone.0067911

29. Narayanan A, Gagliardi F, Gallotti AL, Mazzoleni S, Cominelli M, Fagnocchi L, et al. The Proneural Gene ASCL1 Governs the Transcriptional Subgroup Affiliation in Glioblastoma Stem Cells by Directly Repressing the Mesenchymal Gene NDRG1. Cell Death Differ (2019) 26:1813-31. doi: 10.1038/s41418-018-0248-7 
30. Mazzoleni S, Politi LS, Pala M, Cominelli M, Franzin A, Sergi Sergi L, et al. Epidermal Growth Factor Receptor Expression Identifies Functionally and Molecularly Distinct Tumor-Initiating Cells in Human Glioblastoma Multiforme and is Required for Gliomagenesis. Cancer Res (2010) 70:750013. doi: 10.1158/0008-5472.CAN-10-2353

31. Galli R, Binda E, Orfanelli U, Cipelletti B, Gritti A, De Vitis S, et al. Isolation and Characterization of Tumorigenic, Stem-Like Neural Precursors From Human Glioblastoma. Cancer Res (2004) 64:7011-21. doi: 10.1158/00085472.CAN-04-1364

32. Cominelli M, Grisanti S, Mazzoleni S, Branca C, Buttolo L, Furlan D, et al. EGFR Amplified and Overexpressing Glioblastomas and Association With Better Response to Adjuvant Metronomic Temozolomide. J Natl Cancer Inst (2015) 107(5):djv041. doi: 10.1093/jnci/djv041

33. Reagan-Shaw S, Nihal M, Ahmad N. Dose Translation From Animal to Human Studies Revisited. FASEB J (2008) 22:659-61. doi: 10.1096/fj.079574LSF

34. Terrasi A, Bertolini I, Martelli C, Gaudioso G, Di Cristofori A, Storaci AM, et al. Specific V-ATPase Expression Sub-Classifies IDHwt Lower-Grade Gliomas and Impacts Glioma Growth In Vivo. EBioMedicine (2019) 41:214-24. doi: 10.1016/j.ebiom.2019.01.052

35. Kadota K, Suzuki K, Kachala SS, Zabor EC, Sima CS, Moreira AL, et al. A Grading System Combining Architectural Features and Mitotic Count Predicts Recurrence in Stage I Lung Adenocarcinoma. Mod Pathol (2012) 25:1117-27. doi: 10.1038/modpathol.2012.58

36. Di Grigoli G, Monterisi C, Belloli S, Masiello V, Politi LS, Valenti S, et al. Radiosynthesis and Preliminary Biological Evaluation of [18F]VC701, a Radioligand for Translocator Protein. Mol Imaging (2015) 14. doi: 10.2310/ 7290.2015 .00007

37. Lo Dico A, Martelli C, Valtorta S, Raccagni I, Diceglie C, Belloli S, et al. Identification of Imaging Biomarkers for the Assessment of Tumour Response to Different Treatments in a Preclinical Glioma Model. Eur J Nucl Med Mol Imaging (2015) 42:1093-105. doi: 10.1007/s00259-015-3040-7

38. Garros-Regulez L, Aldaz P, Arrizabalaga O, Moncho-Amor V, CarrascoGarcia E, Manterola L, et al. mTOR Inhibition Decreases SOX2-SOX9 Mediated Glioma Stem Cell Activity and Temozolomide Resistance. Expert Opin Ther Targets (2016) 20:393-405. doi: 10.1517/14728222.2016.1151002

39. Ben-Porath I, Thomson MW, Carey VJ, Ge R, Bell GW, Regev A, et al. An Embryonic Stem Cell-Like Gene Expression Signature in Poorly Differentiated Aggressive Human Tumors. Nat Genet (2008) 40:499-507. doi: $10.1038 /$ ng. 127

40. Garros-Regulez L, Garcia I, Carrasco-Garcia E, Lantero A, Aldaz P, MorenoCugnon L, et al. Targeting SOX2 as a Therapeutic Strategy in Glioblastoma. Front Oncol (2016) 6:222. doi: 10.3389/fonc.2016.00222

41. Yu W, Ren X, Hu C, Tan Y, Shui Y, Chen Z, et al. Glioma SOX2 Expression Decreased After Adjuvant Therapy. BMC Cancer (2019) 19:1087. doi: 10.1186/s12885-019-6292-y

42. Sathyan P, Zinn PO, Marisetty AL, Liu B, Kamal MM, Singh SK, et al. Mir-21Sox2 Axis Delineates Glioblastoma Subtypes With Prognostic Impact. J Neurosci (2015) 35:15097-112. doi: 10.1523/JNEUROSCI.1265-15.2015

43. Politi LS, Brugnara G, Castellano A, Cadioli M, Altabella L, Peviani M, et al. T1-Weighted Dynamic Contrast-Enhanced MRI is a Noninvasive Marker of Epidermal Growth Factor Receptor Viii Status in Cancer Stem Cell-Derived Experimental Glioblastomas. AJNR Am J Neuroradiol (2016) 37:E49-51. doi: 10.3174/ajnr.A4774

44. Yu Z, Zhao G, Li P, Li Y, Zhou G, Chen Y, et al. Temozolomide in Combination With Metformin Act Synergistically to Inhibit Proliferation and Expansion of Glioma Stem-Like Cells. Oncol Lett (2016) 11:2792-800. doi: $10.3892 / \mathrm{ol} .2016 .4315$

45. Lee JE, Lim JH, Hong YK, Yang SH. High-Dose Metformin Plus Temozolomide Shows Increased Anti-Tumor Effects in Glioblastoma in Vitro and In Vivo Compared With Monotherapy. Cancer Res Treat (2018) 50:1331-42. doi: 10.4143/crt.2017.466

46. Emlet DR, Gupta P, Holgado-Madruga M, Del Vecchio CA, Mitra SS, Han SY, et al. Targeting a Glioblastoma Cancer Stem-Cell Population Defined by EGF Receptor Variant Iii. Cancer Res (2014) 74:1238-49. doi: 10.1158/00085472.CAN-13-1407

47. Shinomiya A, Kawai N, Okada M, Miyake K, Nakamura T, Kushida Y, et al. Evaluation of $3^{\prime}$-deoxy-3'-[18F]-fluorothymidine (18f-FLT) Kinetics
Correlated With Thymidine Kinase-1 Expression and Cell Proliferation in Newly Diagnosed Gliomas. Eur J Nucl Med Mol Imaging (2013) 40:175-85. doi: 10.1007/s00259-012-2275-9

48. Lim J, Kim MJ, Park Y, Ahn JW, Hwang SJ, Moon J-S, et al. Upregulation of the NLRC4 Inflammasome Contributes to Poor Prognosis in Glioma Patients. Sci Rep (2019) 9:7895. doi: 10.1038/s41598-019-44261-9

49. Poon CC, Sarkar S, Yong VW, Kelly JJP. Glioblastoma-Associated Microglia and Macrophages: Targets for Therapies to Improve Prognosis. Brain (2017) 140:1548-60. doi: 10.1093/brain/aww355

50. Hambardzumyan D, Gutmann DH, Kettenmann H. The Role of Microglia and Macrophages in Glioma Maintenance and Progression. Nat Neurosci (2016) 19:20-7. doi: 10.1038/nn.4185

51. Liu Q, Tong D, Liu G, Gao J, Wang L, Xu J, et al. Metformin Inhibits Prostate Cancer Progression by Targeting Tumor-Associated Inflammatory Infiltration. Clin Cancer Res (2018) 24:5622-34. doi: 10.1158/1078-0432.CCR-18-0420

52. Foray C, Valtorta S, Barca C, Winkeler A, Roll W, Müther M, et al. Imaging Temozolomide-Induced Changes in the Myeloid Glioma Microenvironment. Theranostics (2021) 11(5):2020-33. doi: 10.7150/thno.47269

53. Gabrusiewicz K, Hossain MB, Cortes-Santiago N, Fan X, Kaminska B, Marini FC, et al. Macrophage Ablation Reduces M2-Like Populations and Jeopardizes Tumor Growth in a MAFIA-Based Glioma Model. Neoplasia (2015) 17:37484. doi: 10.1016/j.neo.2015.03.003

54. Clausi MG, Stessin AM, Zhao Z, Tsirka SE, Ryu S. Neuroinflammatory Changes of the Normal Brain Tissue in Cured Mice Following Combined Radiation and anti-PD-1 Blockade Therapy for Glioma. Sci Rep (2021) 11:5057. doi: 10.1038/s41598-021-84600-3

55. Wang J-C, Sun X, Ma Q, Fu G-F, Cong L-L, Zhang H, et al. Metformin's Antitumour and Anti-Angiogenic Activities are Mediated by Skewing Macrophage Polarization. J Cell Mol Med (2018) 22:3825-36. doi: 10.1111/jcmm.13655

56. Zinnhardt B, Pigeon H, Thézé B, Viel T, Wachsmuth L, Fricke IB, et al. Combined PET Imaging of the Inflammatory Tumor Microenvironment Identifies Margins of Unique Radiotracer Uptake. Cancer Res (2017) 77:1831-41. doi: 10.1158/0008-5472.CAN-16-2628

57. Junck L, Olson JMM, Ciliax BJ, Koeppe RA, Watkins GL, Jewett DM, et al. Pet Imaging of Human Gliomas With Ligands for the Peripheral Benzodiazepine Binding Site. Ann Neurol (1989) 26:752-8. doi: 10.1002/ana.410260611

58. Unterrainer M, Fleischmann DF, Vettermann F, Ruf V, Kaiser L, Nelwan D, et al. Tspo PET, Tumour Grading and Molecular Genetics in Histologically Verified Glioma: A Correlative 18F-GE-180 PET Study. Eur J Nucl Med Mol Imaging (2020) 47:1368-80. doi: 10.1007/s00259-019-04491-5

59. Ferrarese C, Pierpaoli C, Linfante I, Bobo RH, Guthrie B, Kufta C, et al. Peripheral Benzodiazepine Receptors and Glucose Metabolism in Human Gliomas. J Neuro-Oncol (1994) 22:15-22. doi: 10.1007/BF01058351

60. Su Z, Roncaroli F, Durrenberger PF, Coope DJ, Karabatsou K, Hinz R, et al. The 18-Kda Mitochondrial Translocator Protein in Human Gliomas: An 11c(R)Pk11195 PET Imaging and Neuropathology Study. J Nucl Med (2015) 56:512-7. doi: 10.2967/jnumed.114.151621

61. Fu Y, Wang D, Wang H, Cai M, Li C, Zhang X, et al. TSPO Deficiency Induces Mitochondrial Dysfunction, Leading to Hypoxia, Angiogenesis, and a Growth-Promoting Metabolic Shift Toward Glycolysis in Glioblastoma. Neuro-Oncology (2020) 22(2):240-52. doi: 10.1093/neuonc/noz183

62. Seliger C, Luber C, Gerken M, Schaertl J, Proescholdt M, Riemenschneider MJ, et al. Use of Metformin and Survival of Patients With High-Grade Glioma: Metformin and Glioma. Int J Cancer (2019) 144:273-80. doi: $10.1002 /$ ijc. 31783

Conflict of Interest: The authors declare that the research was conducted in the absence of any commercial or financial relationships that could be construed as a potential conflict of interest.

Copyright (๑ 2021 Valtorta, Lo Dico, Raccagni, Martelli, Pieri, Rainone, Todde, Zinnhardt, De Bernardi, Coliva, Politi, Viel, Jacobs, Galli, Ottobrini, Vaira and Moresco. This is an open-access article distributed under the terms of the Creative Commons Attribution License (CC BY). The use, distribution or reproduction in other forums is permitted, provided the original author(s) and the copyright owner(s) are credited and that the original publication in this journal is cited, in accordance with accepted academic practice. No use, distribution or reproduction is permitted which does not comply with these terms. 